\title{
Foreword: sex and sex chromosomes-new clues from nonmodel species
}

\author{
Tariq Ezaz • Jennifer A. Marshall Graves
}

Published online: 25 January 2012

(C) Springer Science+Business Media B.V. 2012

\section{Introduction}

The invitation from the editors Dr Conly Rieder and Prof Herbert Macgregor to edit this issue gave us the opportunity to highlight some biological situations in which sex chromosomes and sex determination are nonstandard, as the result of mutation or capricious evolution. Although they may, indeed, seem weird, these nonmodel species provide us with the genetic variation that can deliver unique, and sometimes surprising, information.

The focus of this issue is, therefore, how much we can learn from such accidents of nature, complemented by variants produced to order. This issue therefore contains articles on sex chromosomes in plants and insects as well as nonmodel vertebrates. The articles contain new data as well as new ideas, hypotheses and opinions, which we hope will attract

Responsible Editor: Conly Rieder

T. Ezaz $(\bowtie)$

Institute for Applied Ecology, University of Canberra,

Canberra, ACT 2601, Australia

e-mail: Traiq.Ezaz@canberra.edu.au

J. A. M. Graves

La Trobe Institute of Molecular Science,

La Trobe University,

Melbourne, VIC 3086, Australia

e-mail: j.graves@latrobe.edu.au

J. A. M. Graves

e-mail: Jenny.Graves@anu.edu.au comment and reaction from readers in other fields. We have gathered articles on sex chromosomes and the genes directly involved in sex determination but also included articles on sex differentiation, including genetic pathways in the development of gonads.

Our other focus is on the extraordinarily rapid progress that can be made using the tools of modern genomics, even in quite bizarre organisms. Each of the systems highlighted in this issue have had their loyal adherents, often over a very long time: for instance, the Y chromosome-less mole vole was first described by Karl Fredga (1970), karyotypes of reptiles were all the rage in the 1970s and bird sex determination has been studied avidly for more than 50 years. However, without access to the armory of molecular techniques, many of these systems have been accessible only at the cytological level.

\section{Sex chromosome organization and evolution}

Sex chromosomes are defined by a sex determining gene or genes, which trigger the genetic pathways that cause undifferentiated gonads to differentiate into either ovary or testes. Depending on whether the sexdetermining gene is male- or female-determining, sex chromosome systems are defined as XX female:XY male (in which the heterogametic $X Y$ male produces $\mathrm{X}$ - or Y-bearing sperm) or ZZ male:ZW female (in which the heterogametic $\mathrm{ZW}$ female produces $\mathrm{Z}$ - or 
W-bearing eggs). The XY system is typified by mammals and fruitflies, but also includes some reptiles and even plants; the ZW system by birds, many reptiles, butterflies, and other plants. Typically, a sex-specific chromosome $(\mathrm{Y}$ or $\mathrm{W})$ is a degraded version of its partner ( $\mathrm{X}$ or $\mathrm{Z})$ which has lost active genes as well as accumulating repetitive DNA (Gschwend et al., Sahara et al., Kawagoshi et al., and Takehana et al., this issue). This characterization of sex chromosomes applies to both plants and animals, and the sex pair shows superficial similarity, composed of one large, gene-rich chromosome, and a degraded version of it. However, the superficial similarity is misleading, for even very similar looking sex pairs (for instance those of birds and snakes) may turn out to be completely nonhomologous, and must have evolved independently (Matsuda et al. 2005). Even some systems in which sex chromosomes show some homology must have been independently derived (Graves and Peichel 2010), suggesting that some chromosomes are good at sex, perhaps because of the conserved genes they bear (O’Meally et al., this issue).

Indeed, one of the hallmarks of sex chromosomes and sex-determining genes is their variety, which is celebrated in this collection. Although the genome is highly conserved between most vertebrates, having essentially the same genes in the same arrangements, sex determination is a remarkable exception. For a trait so fundamental to reproductive fitness and evolutionary success, this seems extraordinary. Yet sex plays by its own evolutionary rules, which are imposed by the very nature of sex determination.

Sex chromosomes are thought to have evolved from an autosomal pair that has acquired a sex-determining gene. Subsequently, other genes with a selective advantage in one sex accumulate within a region that becomes protected from recombination, which then degenerates (Ohno 1967; Graves 2006). Differentiation of mammalian sex chromosomes has occurred through the genetic degradation of the $\mathrm{Y}$ chromosome (Charlesworth 1991, Graves 2006), a process which has gone to different extents in different mammals, even to extinction in two groups of rodents (Murata et al. and Bagheri-Fam et al., this issue). Whether extinction is the fate of all Y chromosomes, including the human $\mathrm{Y}$, was debated at the recent International Chromosome Congress by Jenny Graves and Jenn Hughes (reported by Darren Griffin in this issue). Bird sex chromosomes evolved by the progressive degradation of the $\mathrm{W}$, a process that occurred independently but by similar rules (reviewed by Mank in this issue). In a species of frog, both systems have differentiated independently from the same autosome pair in different populations (Miura et al., this issue). When sex chromosomes undergo rearrangements with autosomes, the translocated autosomal region become subject to the same rules (Tsend-Ayush et al. and Murata et al., in this issue). Thus, specialized sex chromosomes evolve under selective pressures that are different from those that act on autosomes.

Overwhelmingly, studies on sex chromosome evolution and sex determining genes have been performed in humans and mice, and these systems, known in detail, have dramatically improved our understanding of the mechanisms of mammalian sex determination (Koopman et al. 1990; Sinclair et al. 1990; Graves 2002). In comparison, nonmammalian systems are very poorly understood, and only scraps of information are available from other taxa. Yet information from nonmodel animals and plants, which display enormous diversity in sex determination, can be hugely informative, not only about sex chromosome evolution, but also about organization and function.

Of particular interest are reptiles, which display astonishing variation in sex determining systems, including not only every type of sex chromosomes, but also temperature-dependent sex determination and occasionally both (Ezaz et al. 2005; Quinn et al. 2007, Radder et al. 2008). It is remarkable that, despite this diversity, little is known about the genes involved in sex determination in reptiles, and they remain the only vertebrate group in which no master sex determining gene is known. This group promises to yield novel insight into sex determination, particularly agamid lizards and geckos, in which frequent transitions between sex chromosomes systems (XY and ZW) and sex determining modes have been observed among sister species and even within different races of the same species (reviewed Ezaz et al. 2010).

\section{Sex-determining genes}

Despite Herculean efforts, only four vertebrate sex determining genes have been identified. Identification of the master sex determining gene in a particular species is challenging. It requires (1) identifying 
candidate genes and mapping them to the sex chromosomes; (2) demonstrating differential forms or presence/ absence on the $\mathrm{X}$ and $\mathrm{Y}$ (or Z and W) chromosomes; (3) demonstrating differential expression in male and female embryos at an early and critical stage in gonadal development; and (4) experimental manipulation of that expression in vivo to demonstrate a causal link with sexual phenotypes.

All these approaches were used to identify the SRY gene in humans and mice, species in which clinical variants and mutants were available, and to show that it acts as a male dominant to trigger testis differentiation (Koopman et al. 1990; Sinclair et al. 1990). In the absence of sex-reversed variants, the demonstration that sex in the chicken is determined by the dosage of the Z-linked DMRT1 gene, with two copies producing males and one copy producing females, depended more on cytogenetics, comparative genomics, and knockdown experiments (Smith et al. 2009). The other two known genes are both recently retrosposed copies of DMRT1. Sex in Japanese medaka fish is determined by the male-dominant DMY gene that defines a Y chromosome (Matsuda et al. 2002; Nanda et al. 2002), whereas sex in the clawed frog, Xenopus leavis, is determined by the master female-determining gene DMW (possibly male-repressing) that defines a W chromosome (Yoshimoto et al. 2008; Yoshimoto and Ito 2011, reviewed by Mawaribuchi et al., in this issue). How this sex-determining gene could have spawned copies with completely opposite effects is an interesting evolutionary story (Mawaribuchi et al., in this issue).

In mammals, SRY has a partner, the SOX3 gene on the $\mathrm{X}$ chromosome, from which it clearly evolved. This occurred only about 150 million years ago, since SRY is shared by therian mammals, but not monotremes (Graves 2006; Tsend-Ayush et al., in this issue). Mammalian SRY may be nearing the end of its use by date, as demonstrated by two rodent groups that have dispensed with it in favor of as yet unknown substitutes in mole voles (Bagheri-Fam et al., this issue) and spiny rats (Murata et al., this issue). Clearly, there is evidence for many turnovers of the vertebrate sex chromosomes- and sex-determining genes, even among closely related species (Ezaz et al. 2009a, b, 2010; Mawaribuchi et al., this issue), and it is important to understand how one sex-determining gene can give way to another.

\section{Sexual differentiation pathway}

Although gonad differentiation may be triggered by different genes in different animals, the genes on the pathway of sex differentiation are highly conserved at least among vertebrate species, implying a common ancestry. Gonad differentiation is extremely conserved at the histological and the biochemical level, making it informative to compare species as different as alligators and humans.

The genes involved in the sexual differentiation pathway appear to be highly conserved in vertebrates, although homologous genes can sometimes act at different points in the cascade. The genes involved in sexual differentiation have been most thoroughly characterized in humans and mice, by observation of clinical phenotypes and mouse mutations and manipulations (Eggers and Sinclair, this issue). However, interactions are complex, and a number of signaling pathways are involved (Franco and Yao, this issue). Even the wellcharacterized hormone pathways are being reevaluated; the influence of androgens on male phenotype now appears to have an equivalent in estrogen effects on somatic cell phenotype (reviewed by Pask, this issue).

Even for the two best characterized vertebrate sexdetermining genes SRY and DMRT1, fundamental questions remain about how they act to trigger the sex-determining pathway. Although SRY has been known for 20 years, the details of its interaction with SF1 and other factors to trigger the next step in the sex determining pathway, upregulation of the SOX9 gene, are far from clear. Nor is it certain that the gene acts solely through the conserved DNA-binding domain, or is influenced by sequences outside this HMG box (Liang and Koopman, this issue).

Dosage of DMRT1 has been shown experimentally to be critical for male determination in chickens and haploinsufficiency of this gene is associated with sex reversal in humans (Ferguson-Smith 2007). In addition, mutations of autosomal DMRT1 in medaka fishes also found to cause functional sex reversal in Japanese medaka fish (Masuyama et al., this issue). However, the pathway triggered by DMRT1 dosage is also complex. Evidence from gyandromorph chickens mandates another cell-autonomous factor that determines somatic phenotype (Clinton et al., this issue), and it is likely that micro-RNAs are involved (Cutting et al., this issue). 


\section{Potential of new technology}

The introduction of new cytogenetic and genomic technologies, and the availability of sequence information for many species, has radically changed the prospects for discovery and characterization of sex-determining genes in nonmodel species.

Now, it is possible to unite molecular cytogenetics and comparative genomics to make direct studies of the sex chromosomes and the genes they bear in any species, no matter how bizarre. For instance, starting with only crude cytology of the dragon lizard Pogona vitticeps a few years ago, we could identify cryptic sex chromosomes using comparative genome hybridization (Ezaz et al. 2005), allowing us to physically isolate the $\mathrm{Z}$ and $\mathrm{W}$ by microdissection, prepare Z- and W-specific DNA and use it to identify the region of the conserved bird-reptile genome that is concerned with sex in this species, hopefully identifying candidate sex-determining genes.

Next-generation sequencing provides opportunities to sequence whole genomes from various nonmodel species. We hope, particularly, that this will include many reptiles, only one species of which has so far been sequenced. We welcome the proposal by the Genome $10 \mathrm{~K}$ consortium to sequence every vertebrate (Genome 10K 2009), and we urge the sequencing of animals of both sex (particularly for species in which it is not clear even which sex is heterogametic), or targeted sequencing of physically isolated sex chromosomes.

Next-generation sequencing can also be used to explore the influence of epigenetic variation, as well as small RNA, in sex determination and dosage compensation in many species, particularly in reptile and fish species in which sex is environmentally determined. An exciting and challenging phase is ahead in which the genetic variation offered by nonmodel species can yield unique information on a par of sophistication with the well-studied human, mouse, and fruitfly.

Acknowledgments Putting such a varied and interesting volume together required all the contacts and resources borne of our varied experience in sex in fish (Ezaz) and mammals (Graves), and our close collaboration on reptile and amphibian sex. We are very grateful for the active and consistent support and encouragement from editors Conly Rieder and Herbert Macgregor. We thank them in particular for humoring our choice of what may seem a fairly bizarre set of systems to explore, and for encouraging us to seek contributions from emerging young scientists. Above all, we thank all the authors who agreed to contribute their exciting new research findings, opinions and reviews. We also appreciate time and constructive suggestions from all our reviewers, which greatly enhanced the quality of this compendium.

\section{References}

Charlesworth B (1991) The evolution of sex chromosomes. Science 251:1030-1033

Ezaz T, Quinn AE, Miura I, Sarre SD, Georges A, Marshall Graves JA (2005) The dragon lizard Pogona vitticeps has ZZ/ZW micro-sex chromosomes. Chromosome Res 13:763-776

Ezaz T, Quinn AE, Sarre SD, O’Meally D, Georges A, Graves JAM (2009a) Molecular marker suggests rapid changes of sex-determining mechanisms in Australian dragon lizards. Chromosome Res 17:91-98

Ezaz T, Moritz B, Waters PD, Graves JAM, Georges A, Sarre SD (2009b) The ZW sex microchromosomes of an Australian dragon lizard share no homology with those of other reptiles or birds. Chromosomes Res 17:965-973

Ezaz T, Sarre SD, O’Meally, Graves JAM, Georges A (2010) Sex chromosome evolution in lizards: independent origins and rapid transitions. Cytogenet and Genome Res 127:249-260

Ferguson-Smith M (2007) The evolution of sex chromosomes and sex determination in vertebrates and the key role of DMRT1. M Sex Dev 1:2-11

Fredga K (1970) Unusual sex chromosome inheritance in mammals. Phil Trans R SOC Lond Ser B 259:15-36

Genome 10K Community of Scientists (2009) A proposal to obtain whole genome sequence for 10,000 vertebrate species. J Hered 100:659-674

Graves JAM (2002) Sex chromosomes and sex determination in weird mammals. Cytogenet and Genome Res 96:161-168

Graves JAM (2006) Sex chromosome specialization and degeneration in mammals. Cell 124:901-914

Graves JAM, Peichel K (2010) Are homologies in vertebrate sex determination due to shared ancestry or to limited options? Genome Biol 11:205

Koopman P, Munsterberg A, Capel B, Vivian N, Lovell-Badge R (1990) Expression of a candidate sex determining gene during mouse testis differentiation. Nature 348:450-452

Matsuda M, Nagahama Y, Shinomiya A, Sato T, Matsuda C, Kobayashi T, Morrey CE, Shibata N, Asakawa S, Shimizu N, Hori H, Hamaguchi S, Sakaizumi M (2002) DMY is a Y-specific DM-domain gene required for male development in the medaka fish. Nature 417:559-563

Matsuda Y, Nishida-Umehara C, Tarui H, Kuroiwa A, Yamada $\mathrm{K}$, Isobe $\mathrm{T}$ et al (2005) Highly conserved linkage homology between birds and turtles: bird and turtle chromosomes are precise counterparts of each other. Chromosome Res 13:601-615

Nanda I, Kondo M, Hornung U, Asakawa S, Winkler C, Shimizu A, Shan Z, Haaf T, Shimizu N, Shima A, Schmid M, Schartl M (2002) A duplicated copy of DMRT1 in the sex- 
determining region of the Y chromosome of the medaka, Oryzias latipes. PNAS USA 99:11778-11783

Ohno S (1967) Sex chromosomes and sex-linked genes. Springer, Heidelberg

Quinn AE, Georges A, Sarre SD, Guarino F, Ezaz T, Graves JA (2007) Temperature sex reversal implies sex gene dosage in a reptile. Science 316:411

Radder RS, Quinn AE, Georges A, Sarre SD, Shine R (2008) Genetic evidence for co-occurrence of chromosomal and thermal sex-determining systems in a lizard. Biol Lett 4:176-178

Sinclair AH, Berta P, Palmer MS, Hawkins JR, Griffiths BL, Smith MJ (1990) A gene from the human sex-determining region encodes a protein with homology to a conserved DNA-binding motif. Nature 346:240-244

Smith CA, Roeszler KN, Ohnesorg T, Cummins DM, Farlie PG, Doran TJ et al (2009) The avian Z-linked gene dmrt1 is required for male sex determination in the chicken. Nature 461:267-271

Yoshimoto S, Ito M (2011) A ZZ/ZW-type sex determination in Xenopus laevis. FEBS J 278:1020-1026

Yoshimoto S, Okada E, Umemoto H, Tamura K, Uno Y, Nishida-Umehara C et al (2008) A W-linked dm-domain gene, DM-W, participates in primary ovary development in Xenopus laevis. PNAS USA 105:2469-2474 